\title{
Estimating the Cost of Construction of Light Water Reactor Plants Using Multiple Regression Model
}

\author{
Rami H. Alamoudi ${ }^{1}$ \\ ${ }^{\text {I}(D e p a r t m e n t ~ o f ~ I n d u s t r i a l ~ E n g i n e e r i n g, ~ K i n g ~ A b d u l a z i z ~ U n i v e r s i t y, ~ J e d d a h, ~ S a u d i ~ A r a b i a) ~}$
}

\begin{abstract}
This paper is an approach to estimate the cost of construction of light water reactor (LWR) plants using information about previous research. A number of variables that are expected to be effective in predicting this cost have been selected. The multiple regression models for the estimation of the cost of construction of light water reactor (LWR) plants using the set of predictors have been developed. The nuclear data frame has 32 rows and 11 columns which mean that we have 10 independent variables and 32 samples. The outcome of the regression analysis shows that the Cost of a future power plant can be estimated using the following independent variables: The date on which the construction permit was issued. The data are measured in years since January 11990 to the nearest month. The net capacity of the power plant (MWe). The location of the power plant in the US (If the Plant was constructed in the north-east region of USA). In the end of the paper the equation of estimating the cost of a future power plant has been developed.
\end{abstract}

Keywords - Light Water Rector, Matrix Plot, Multiple Regressions, Outlier Test, Stepwise Regression,

\section{INTRODUCTION}

The search for an alternative source of power other than the fossil based fuels; not only for economical and political reasons but also for environmental reasons; have increased the needs to Construct light water reactor (LWR) plants in the US [1] . The costs related to the construction of light water reactor (LWR) plants started getting a great deal of attention in the late 1960's and early 1970's , due to the future plans of construction and renewal of more (LWR) plants throughout the US. It was important to try to predict the capital cost of construction and renewal of more (LWR) plants, and through this process, some questions started to arise, what are the factors affecting the capital cost of construction?, some important factors that might have an effect on the capital cost of construction as follow:

\section{Date factor :}

a. The date on which the construction permit was issued

2. Time Factors :

a. The time between application for and issue of the construction permit

b. The time between issue of operating license and construction permit

3. Capacity Factor :

a. The net capacity of the power plant (MWe).

\section{Location Factors :}

a. The prior existence of a LWR plant at the same site.

b. If the Plant was constructed in the north-east region of USA

5. Technical factors :

a. If a cooling tower is present in the plant

6. Construction factors :

a. If the nuclear steam supply system was manufactured by Babcock-Wilcox.

b. The cumulative number of power plants constructed by each architect-engineer.

c. If plants are with partial turnkey guarantees. (some of the power plants had partial turnkey guarantees and it is possible that, for these plants, some manufacturers' subsidies may be hidden in the quoted capital costs)

In this paper, we investigate the effect of each of those factors on the overall capital cost of construction and to use their conclusions for future prediction of the costs related to the construction of light water reactor (LWR) plants in the future. 


\section{RESEARCH METHODOLOGY}

Based on their preliminary researches [2,3] on the factors affecting the costs related to the construction of light water reactor (LWR) plants, we have started collecting data related to the construction of 32 light water reactor (LWR) plants constructed in the US in the late 1960's and early 1970's [1]. The nuclear data frame has 32 rows and 11 columns and contains the following columns:

cost: The capital cost of construction in millions of dollars adjusted to 1976 base.

date: The date on which the construction permit was issued. The data are measured in years since January 11990 to the nearest month.

t1: The time between application for and issue of the construction permit.

t2: The time between issue of operating license and construction permit.

cap: The net capacity of the power plant (MWe).

pr: A binary variable where 1 indicates the prior existence of a LWR plant at the same site.

ne: A binary variable where 1 indicate that plant was constructed in the north-east region of USA

ct: A binary variable where 1 indicates the use of a cooling tower in the plant.

bw: A binary variable where 1 indicates that the nuclear steam supply system was manufactured by Babcock-Wilcox.

cum.n: The cumulative number of power plants constructed by each architect-engineer.

pt: A binary variable where 1 indicates those plants with partial turnkey guarantees.

The first step was to try to evaluate the explanatory power of the variables on the Cost, and also try to detect redundant variables or multi co-linearity among the independent variables. This was to reach the optimum valid model with the best predictors of cost.

A preliminary regression analysis was performed to assess all that, through a matrix plot (a plot showing predictors vs predicted and predictors vs one another) and Residual vs fitted values plot [4]. We can notice two problems, problem of non constant variance in the residual plots, so transformation of the predicted Variable (cost) was needed to overcome this problem. And a non linear relationship between Cost and the cumulative number of power plants constructed by an engineer ( Cum.n ), So we need to standardized the data and try for a transformation of cum.n too and we tried several transforms ( monitoring the significance of the partial T-test). The results were $b$ that the best transform to eliminate the non-constant variance was $1 / \ln ($ cost) and to include cum.n, cum. $\mathrm{n}^{\wedge} 2$, Cum. $\mathrm{n}^{\wedge} 3$ in the model was the only way to get a significant linear relationship for the variable cum.n.

The next Phase was getting rid of redundant variables (like t2; having no relationship with $1 / \ln$ (cost) and multicolinearity among variables ( like the case between Date and t1 ) and reducing the variables only the most significant and with the highest explanatory power using stepwise technique [5].

Through regressions and evaluation of the partial t-tests, residual plots and sequential sum of squares and other methods like best subset and stepwise regressions, we ended up with three most significant variables $[6,7]$. They showed random residual plots, partial t-tests with p-values of zero, reasonable ssq and a variance inflation factor of one. The only problem was two outliers in the residual vs fitted values plot that didn't show in the residual plot of the variables, which suggested the presence of influential outliers, so we performed some outliers tests like cooks distance, hat matrix and DFITS they all showed the same two outliers ( they were the same outliers on the residual vs fitted value graph ), so going back to the data sources, we were able to eliminate those two points and we ended up with a reasonable model for predicting the Cost.

\section{RESEARCH ANALYSIS}

Looking for directly correlated variables, we decided to perform a multiple linear regression run, and check the Matrix plot (Fig. 1), and since the matrix plot doesn't give enough information about binary variables, we only plotted the quantitative variables.

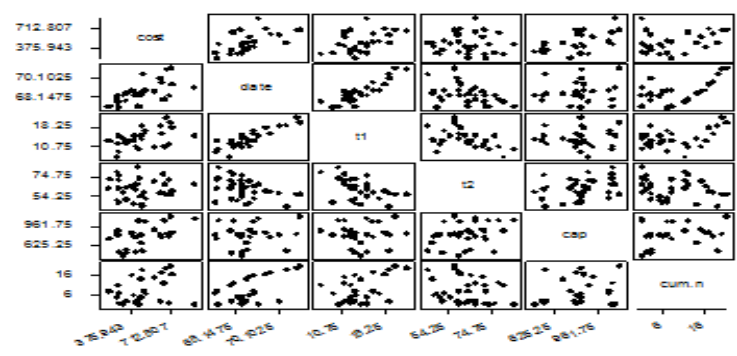

Fig. 1 The matrix plot for the preliminary regression model 
The Preliminary Regression Observations will be as follow: The matrix plot and normal probability and residual plots will be discussed as follow:

1- Matrix plot :

a. Predicted vs Predictors :

i.Cost vs Date : Looks like a Linear relation ..fine

ii.Cost vs $\mathrm{t} 1$ : Looks like a Linear relation ..fine

iii.Cost vs $\mathrm{t} 2$ : Looks completely random ..suggests no relationship

iv. Cost vs Cap : Looks like a Linear relation ..fine

v. Cost vs Cum.n : Looks like a non-Linear relation .. (U shaped), might need transformation.

b. Predictors vs Predictors :

i. Date vs t1 : Looks like a Linear relation...eliminate one of them (later we will see that we chose t1)

ii. Date vs $\mathrm{t} 2$ : Looks like an inverse Linear relation...might eliminate one of them ( later we will see that we chose $\mathrm{t} 2$ )

iii. Date vs Cap : looks random

iv. Date vs cum.n : Looks like a Linear relation .

2- Normal probability and residual plots :

a. The normal probability plot looks very good

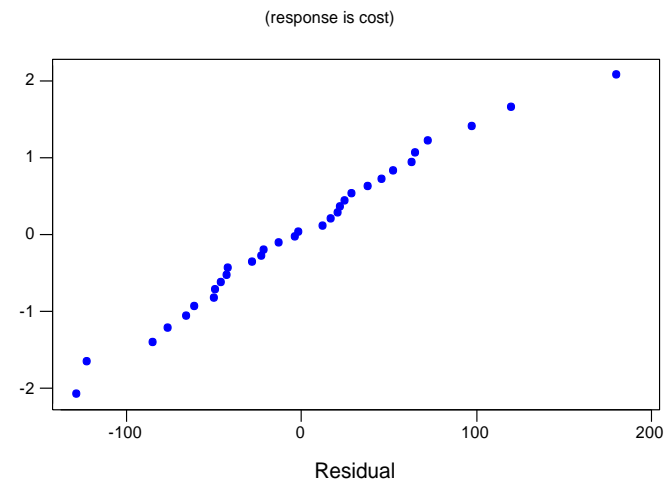

Fig. 2 Normal probability plot of the residual for the preliminary regression model

b. Residuals vs fitted values ...looks like there is some funneling there ...with may be one outlier. So a transformation might be recommended to avoid the non-constant variance (that shows in the funneling).

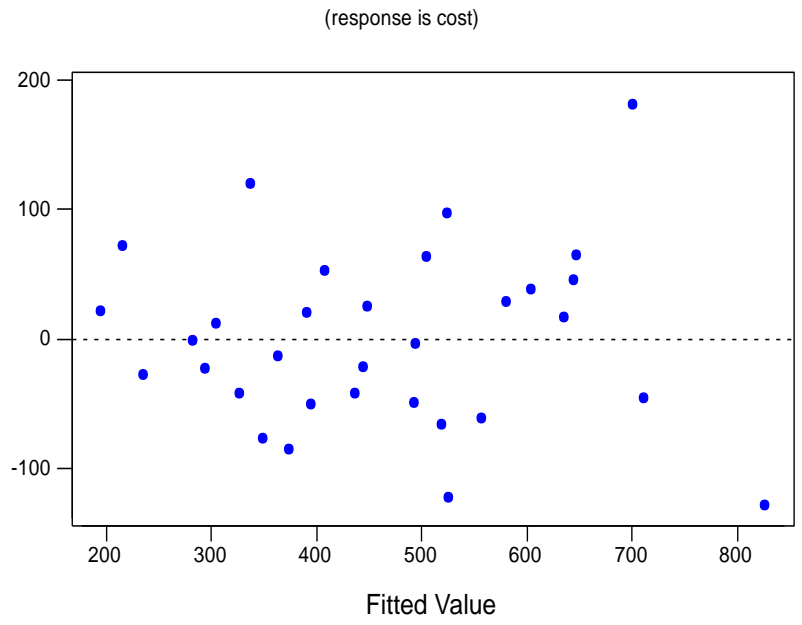

Fig. 3 Residual versus the fitted values for the preliminary regression model

c. Residuals vs Date random with a couple of outliers maybe. 


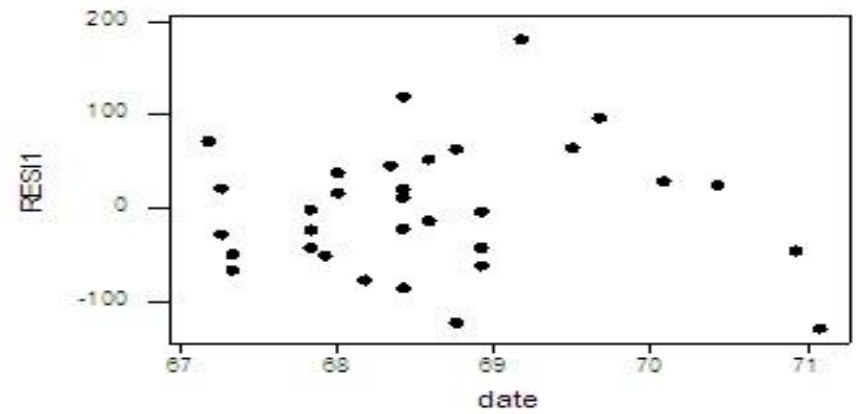

Fig. 4 Residuals vs Date random for the preliminary regression model

d. Residuals vs $\mathrm{t} 1$ random

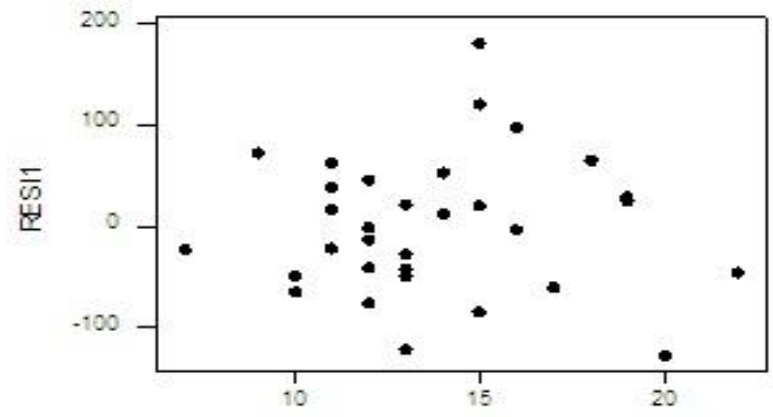

t1

Fig. 5 Residuals vs t1 random for the preliminary regression model

e. Residuals vs $\mathrm{t} 2$ random

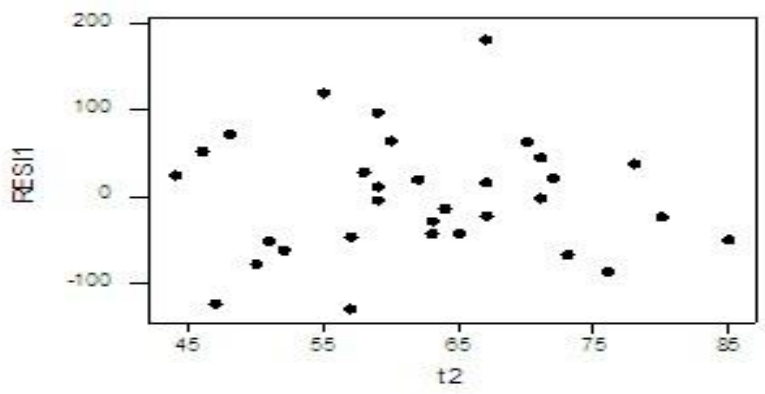

Fig. 6 Residuals vs $\mathrm{t} 2$ random for the preliminary regression model

f. Residuals vs Cum.n, "U “ shaped.. support the transformation decision

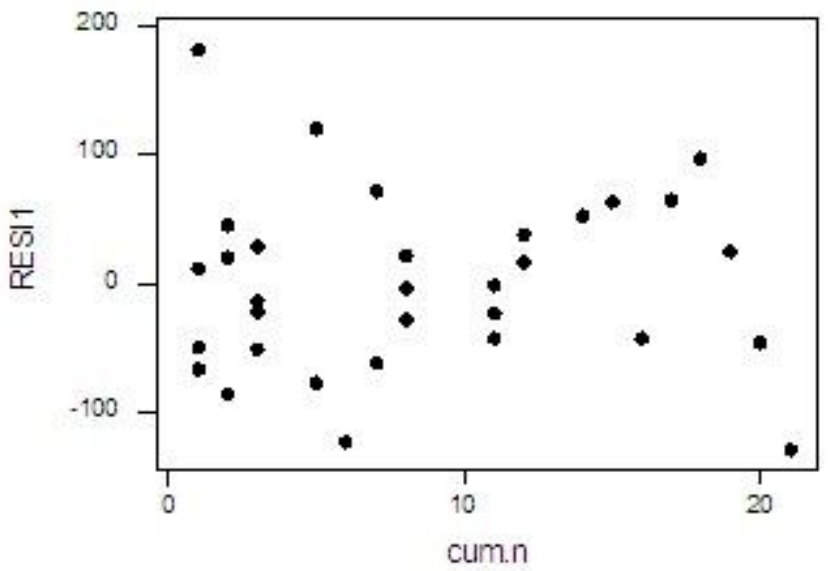

Fig. 7 Residuals vs Cum.n random for the preliminary regression model 
From the above analysis, we have non constant variance clear in the funneling of the residual verses fit plot, thus we need to search for a proper transform.

After a number of trials to transform Cost and checking for the randomness of the residual versus fitted value plot, and also looking at the matrix plot, we ended up choosing the $1 / \ln (\operatorname{cost})$, as the best possible transform. [8].

It was noted earlier that the variable cum.n appeared to have a non linear relationship with the cost, this non-linear relationship stayed after transformation but the variable stayed statistically insignificant, so I decided to include the variable cum. $n^{\wedge} 2$ and standardize but still. So we included also cum. $n^{\wedge} 3$ and standardized and now we got them significant [9]

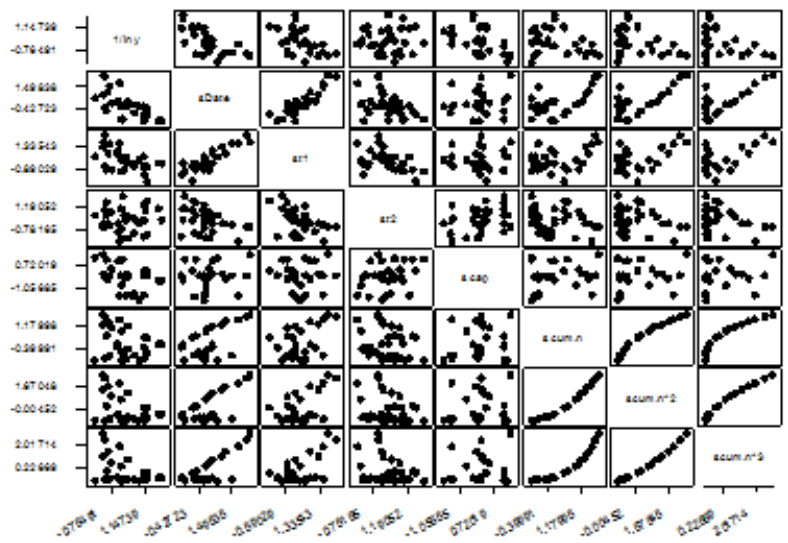

Fig. 8 The matrix plot after transformation

Thus, reapplying the matrix plot and Normal probability and residual plots as follow:

\section{Matrix plot :}

Predicted vs Predictors :

I. $s(1 /$ lncost $)$ vs sDate : Looks like a -ve Linear relation ..fine

II. $s(1 /$ lncost $)$ vs st 1 : Looks like a-ve Linear relation ..fine

III. s(1/lncost) vs st 2 : Looks completely random ..suggests no relationship

IV. $s(1 /$ lncost $)$ vs sCap : Looks like a-ve Linear relation ..fine

V. s(1/lncost) vs Cum.n cumn^2 and cumn^3: Looks like a -ve Linear relation ..fine

Predictors vs Predictors :

VI. sDate vs st1 : Looks like a Linear relation...eliminate one of them ( I Choose st1)

VII. sDate vs st2 : Looks like an inverse Linear relation...might eliminate one of them

VIII. sDate vs sCap : looks random

IX. sDate vs sCum.n scumn^2 and scumn^3 : Looks like a Linear relations there

X. st1 vs sCum.n scumn^2 and scumn^3 : Looks like a Linear relations there

The Normal probability and residual plots (After Transformation) will be as shown in Fig. 9.
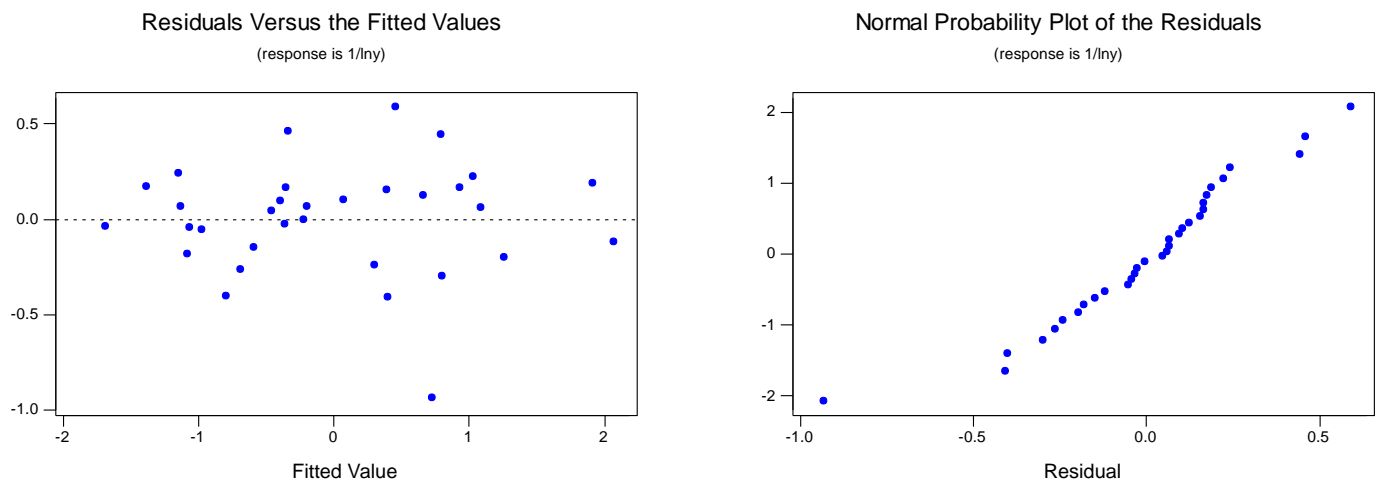

Fig. 9 Normal probability and residual vs fitted values plots after transformation 
The normal probability plot has an obvious outlier there. Residuals vs fitted values ...looks like there is no funneling now...but there might be one outlier. The Regression Analysis after Transformation will be as follow:

The regression equation is:

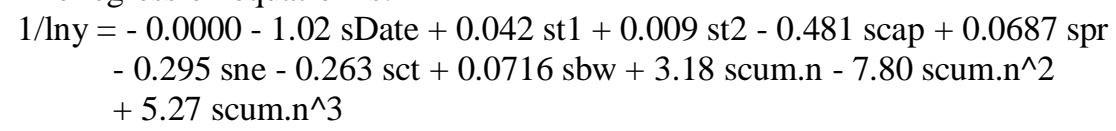

\begin{tabular}{|c|c|c|c|}
\hline Predictor & Coef & StDev & $\mathrm{T}$ \\
\hline Constant & -0.00000 & 0.06359 & -0.00 \\
\hline sDate & -1.0230 & 0.1761 & $\begin{array}{ll}-5.81 & 0.000\end{array}$ \\
\hline st1 & 0.0421 & 0.1573 & $0.27 \quad 0.792$ \\
\hline st2 & 0.0087 & 0.1276 & $0.07 \quad 0.946$ \\
\hline scap & -0.48129 & 0.07592 & $\begin{array}{ll}-6.34 & 0.000\end{array}$ \\
\hline spr & 0.06866 & 0.09324 & $0.74 \quad 0.470$ \\
\hline sne & -0.29468 & 0.06942 & 0.000 \\
\hline sct & -0.26255 & 0.07984 & 0.004 \\
\hline sbw & 0.07159 & 0.08800 & 0.426 \\
\hline scum.n & 3.1803 & 0.8867 & 0.002 \\
\hline scum. $n^{\wedge}$ & -7.795 & 2.021 & 0.001 \\
\hline scum. $n^{\wedge}$ & 5.267 & 1.246 & 0.000 \\
\hline
\end{tabular}

$\mathrm{S}=0.3597 \quad \mathrm{R}-\mathrm{Sq}=91.7 \% \quad \mathrm{R}-\mathrm{Sq}(\mathrm{adj})=87.1 \%$

Analysis of Variance

\begin{tabular}{|c|c|c|c|c|c|c|}
\hline \multicolumn{2}{|l|}{ Source } & DF & SS & MS & $\mathrm{P}$ & \\
\hline \multicolumn{2}{|c|}{ Regression } & 11 & 28.4120 & 2.5829 & 19.96 & 00 \\
\hline \multicolumn{2}{|c|}{ Residual Error } & 20 & 2.5880 & 0.1294 & & \\
\hline Total & 3 & \multicolumn{2}{|c|}{131.0000} & & & \\
\hline Source & DF & \multicolumn{2}{|c|}{ Seq SS } & & & \\
\hline sDate & 1 & \multicolumn{2}{|c|}{12.2940} & & & \\
\hline st1 & 1 & \multicolumn{2}{|c|}{1.0097} & & & \\
\hline st2 & 1 & \multicolumn{2}{|c|}{1.0150} & & & \\
\hline scap & 1 & \multicolumn{2}{|c|}{3.7406} & & & \\
\hline spr & 1 & \multicolumn{2}{|c|}{1.0806} & & & \\
\hline sne & 1 & \multicolumn{2}{|c|}{3.5404} & & & \\
\hline sct & 1 & \multicolumn{2}{|c|}{0.9954} & & & \\
\hline sbw & 1 & \multicolumn{2}{|c|}{0.3975} & & & \\
\hline & & \multicolumn{2}{|c|}{1.8744} & & & \\
\hline \multicolumn{4}{|c|}{$\begin{array}{lll}\text { scum.n } n^{\wedge} 2 & 1 & 0.1515\end{array}$} & & & \\
\hline \multicolumn{2}{|l|}{ scum.n^3 } & \multicolumn{2}{|c|}{2.3129} & & & \\
\hline \multicolumn{7}{|c|}{ Unusual Observations } \\
\hline Obs $\quad \mathrm{sD}$ & Date & $1 / \ln$ & Fit & StDev Fit & Residual & St Resid \\
\hline-0.1 & & -0.209 & 0.7247 & 0.1729 & -0.9337 & $-2.96 \mathrm{R}$ \\
\hline 18 & 16 & 1.048 & 0.4590 & 0.2235 & 0.5893 & $2.09 \mathrm{R}$ \\
\hline
\end{tabular}

We can observe the following:

1. Over All F-test :

$\mathrm{HO}:$ all $\mathrm{Bi}=$ zero

$\mathrm{Ha}:$ at least one $\mathrm{Bi}$ not $=$ zero

p-value $=0$, Reject the null hypothesis

Therefore at least one Bi not equal to zero. The model stands.

2. Predictors :

Best Subsets Regression: 1/lny versus sdate, st1, ... 
Response is $1 /$ lny

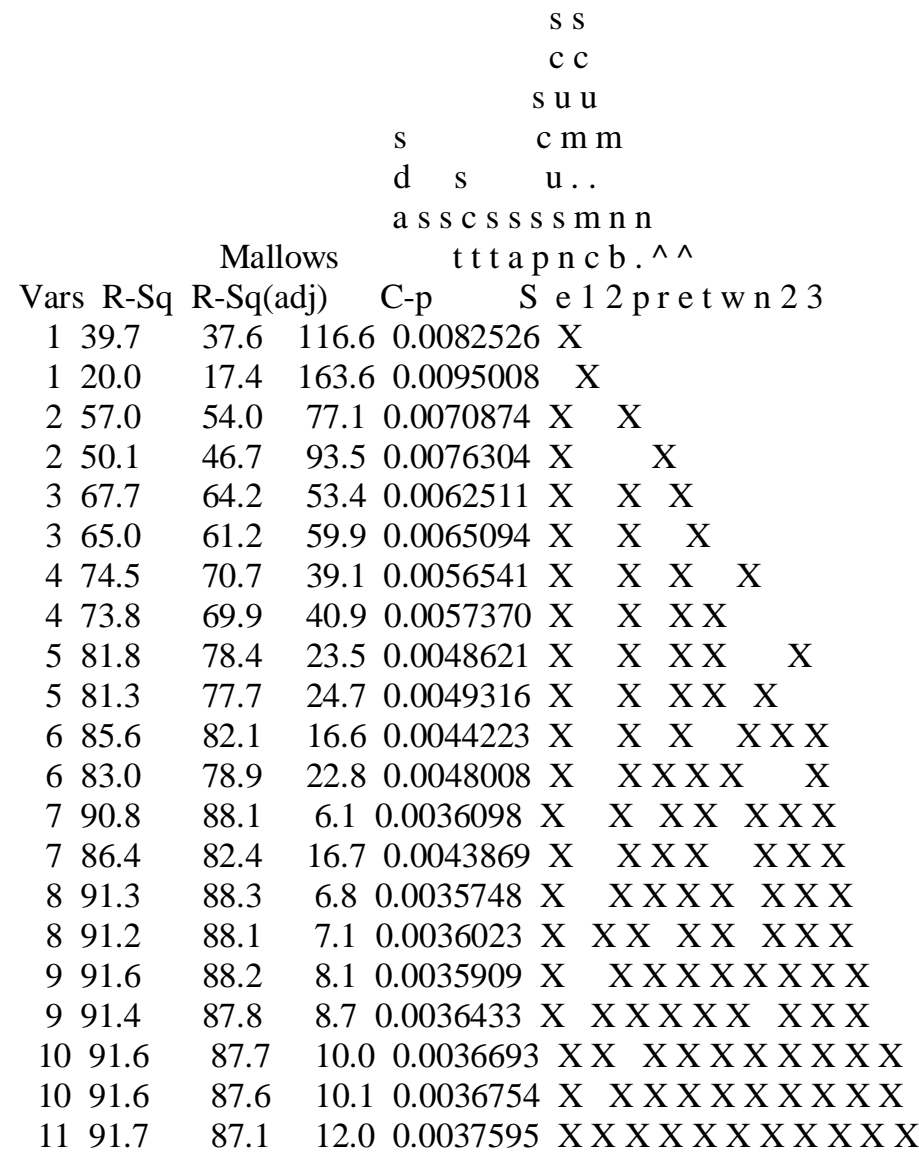

Stepwise Regression: 1/lny versus sdate, st $1, \ldots$

Alpha-to-Enter: 0.15 Alpha-to-Remove: 0.15

Response is $1 /$ lny on 11 predictors, with $\mathrm{N}=32$

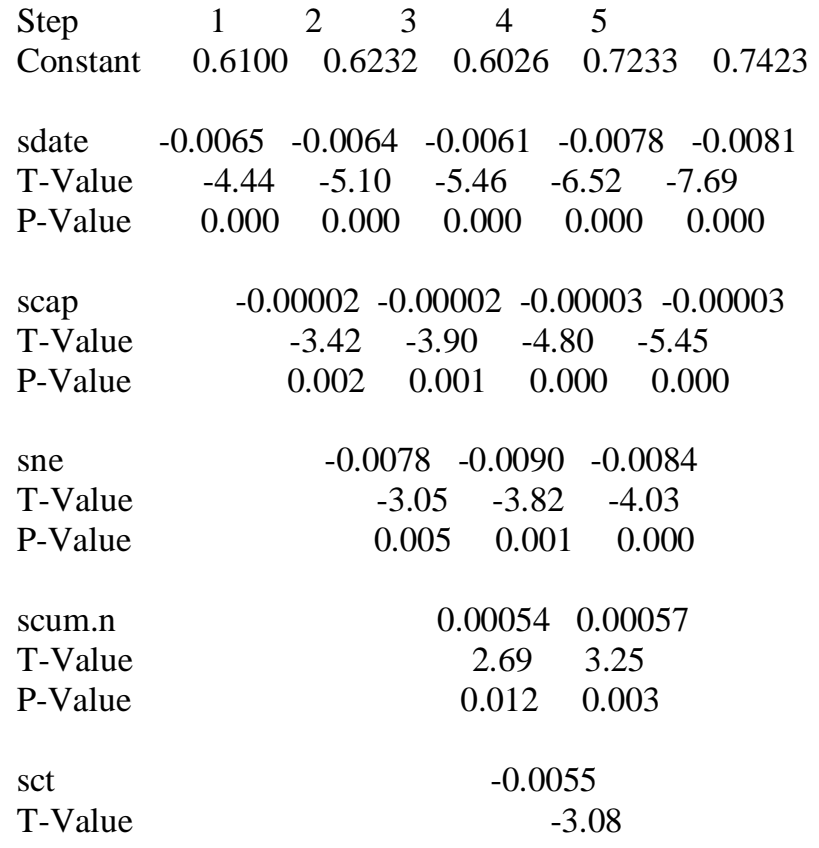


P-Value

0.005

$\begin{array}{lccccc}\mathrm{S} & 0.00825 & 0.00709 & 0.00625 & 0.00565 & 0.00493 \\ \text { R-Sq } & 39.66 & 56.98 & 67.69 & 74.51 & 81.32 \\ \text { R-Sq(adj) } & 37.65 & 54.01 & 64.22 & 70.73 & 77.73 \\ \text { Mallows C-p } & 116.6 & 77.1 & 53.4 & 39.1 & 24.7\end{array}$

Looking at the Stepwise Regression, Best Subsets Regression and the partial T-test P-value of each predictor, and its corresponding seq ss [9]. We can say that:

- sdate, scap, sne, sCum.n scumn^2 and scumn^3 are all significant with p-value $<0.05$ But

- since the matrix plot showed linear relation between Sdate and sCum.n scumn^2 and scumn^3 ,And also linear relation between Sdate and st1, we will try eliminating st1, sCum.n, scumn^${ }^{\wedge} 2$ and scumn^3 from my model.

- st2 with p-value > 0.05 and no relationship with $\mathrm{s}(1 / \ln (\operatorname{cost}))$, so we decided to eliminate it from the model.

The run after First Reduction, after eliminating st1, scum.n, scumn^ 2 and scumn ${ }^{\wedge} 3$ and st 2 we get the following:

The regression equation is

$$
\begin{aligned}
1 / \mathrm{lny} & =-0.0000-0.590 \text { sDate }-0.432 \mathrm{scap}+0.090 \mathrm{spr}-0.292 \mathrm{sne}-0.231 \mathrm{sct} \\
& +0.050 \mathrm{sbw}
\end{aligned}
$$

Predictor Coef StDev $\mathrm{T} \quad \mathrm{P}$

Constant $\quad-0.00000 \quad 0.09893 \quad-0.00 \quad 1.000$

$\begin{array}{lllll}\text { sDate } & -0.5905 & 0.1027 & -5.75 & 0.000\end{array}$

$\begin{array}{lllll}\text { scap } & -0.4322 & 0.1028 & -4.20 & 0.000\end{array}$

$\begin{array}{lllll}\text { spr } & 0.0903 & 0.1035 & 0.87 & 0.391\end{array}$

$\begin{array}{lllll}\text { sne } & -0.2917 & 0.1021 & -2.86 & 0.008\end{array}$

$\begin{array}{lllll}\text { sct } & -0.2314 & 0.1028 & -2.25 & 0.033\end{array}$

$\begin{array}{lllll}\text { sbw } & 0.0498 & 0.1032 & 0.48 & 0.634\end{array}$

$\mathrm{S}=0.5596 \quad \mathrm{R}-\mathrm{Sq}=74.7 \% \quad \mathrm{R}-\mathrm{Sq}(\mathrm{adj})=68.7 \%$

\begin{tabular}{|c|c|c|c|c|c|c|}
\hline \multicolumn{2}{|c|}{ Source } & $\mathrm{DF}$ & SS & \multirow{2}{*}{$\begin{array}{l}\text { MS } \\
3.8616\end{array}$} & \multirow{2}{*}{$\begin{array}{l}F_{12.33} P \\
\end{array}$} & \\
\hline \multirow{2}{*}{\multicolumn{2}{|c|}{$\begin{array}{l}\text { Regression } \\
\text { Residual Frro }\end{array}$}} & 6 & 23.1698 & & & 0.000 \\
\hline & & 2 & 7.8302 & 0.3132 & & \\
\hline \multicolumn{2}{|c|}{ Total 3} & 31 & 31.0000 & & & \\
\hline Source & DF & & eq SS & & & \\
\hline sDate & 1 & & 2940 & & & \\
\hline scap & 1 & 5.3 & 694 & & & \\
\hline spr & 1 & 0.65 & & & & \\
\hline sne & 1 & 3.12 & & & & \\
\hline sct & 1 & 1.65 & & & & \\
\hline sbw & 1 & & 729 & & & \\
\hline
\end{tabular}

Analysis of Variance

Unusual Observations

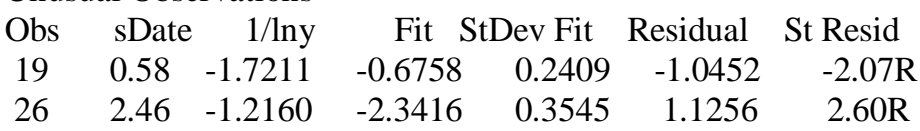

Best Subsets Regression: 1/lny versus sdate, scap, spr, sne, sct, sbw Response is $1 /$ lny

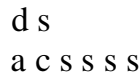




\begin{tabular}{|c|c|c|c|c|}
\hline \multicolumn{3}{|c|}{ Mallows } & \multicolumn{2}{|c|}{ tapncb } \\
\hline Vars R-Sq & $\mathrm{R}-\mathrm{Sq}$ & & C-p $\quad$ S & $S$ e pretw \\
\hline 139.7 & 37.6 & 31.7 & 0.0082526 & $6 x$ \\
\hline 118.4 & 15.6 & 52.8 & 0.0095993 & $3 x$ \\
\hline 257.0 & 54.0 & 16.6 & 0.0070874 & $4 \times X$ \\
\hline 250.1 & 46.7 & 23.4 & 0.0076304 & $4 \mathrm{X} \quad \mathrm{X}$ \\
\hline 367.7 & 64.2 & 8.0 & 0.0062511 & XX $X$ \\
\hline 365.0 & 61.2 & 10.7 & 0.0065094 & $4 \times X \quad X$ \\
\hline 473.8 & 69.9 & 4.0 & 0.0057370 & XX XX \\
\hline 469.2 & 64.6 & 8.5 & 0.0062167 & X X X X \\
\hline 574.5 & 69.6 & 5.2 & 0.0057620 & XXXXX \\
\hline 574.0 & 69.0 & 5.8 & 0.0058222 & $X X X X X$ \\
\hline & & & & \\
\hline
\end{tabular}

Stepwise Regression: 1/lny versus sdate, scap, spr, sne, sct, sbw

Alpha-to-Enter: 0.15 Alpha-to-Remove: 0.15

Response is $1 /$ lny on 6 predictors, with $\mathrm{N}=32$

\begin{tabular}{|c|c|c|c|c|}
\hline Step & 1 & 23 & 4 & \\
\hline Constant & 0.6100 & 0.6232 & 0.6026 & 0.6143 \\
\hline sdate & -0.0065 & -0.0064 & -0.0061 & -0.0062 \\
\hline T-Value & -4.44 & -5.10 & -5.46 & -6.08 \\
\hline P-Value & 0.000 & 0.000 & 0.000 & 0.000 \\
\hline scap & -0.0 & $00002-0$ & $.00002-0$ & 0.00002 \\
\hline T-Value & & -3.42 & $-3.90 \quad-4$ & 4.17 \\
\hline P-Value & & 0.002 & 0.001 & 0.000 \\
\hline sne & \multicolumn{4}{|c|}{$-0.0078 \quad-0.0071$} \\
\hline T-Value & \multicolumn{4}{|c|}{$\begin{array}{ll}-3.05 & -3.01\end{array}$} \\
\hline P-Value & \multicolumn{4}{|c|}{0.005} \\
\hline sct & \multicolumn{4}{|c|}{-0.0052} \\
\hline T-Value & \multicolumn{4}{|c|}{-2.50} \\
\hline P-Value & \multicolumn{4}{|c|}{0.019} \\
\hline $\mathrm{S}$ & 0.00825 & 0.00709 & 0.00625 & 0.00574 \\
\hline $\mathrm{R}-\mathrm{Sq}$ & 39.66 & 56.98 & 67.69 & 73.76 \\
\hline $\mathrm{R}-\mathrm{Sq}(\mathrm{adj})$ & 37.65 & 54.01 & 64.22 & 69.87 \\
\hline Mallows & C-p 31. & 16.6 & 8.0 & 4.0 \\
\hline
\end{tabular}

Looking at the Stepwise Regression, Best Subsets Regression and the partial T-test P-value of each predictor, and its corresponding seq ss. We can see that spr, sct, sbw, and spt are all with p-value $>0.05$ and with small contribution in the seq ss. So we decide to REMOVE ct, bw , pr and pt , from the model, and run it again. The run after second Reduction of variables [10], now we ran only the variables Date, Cap and ne against the $1 / \ln (\cos t)$

The regression equation is

$1 /$ ln $y=0.603-0.00607$ date -0.000023 cap -0.00781 ne

$\begin{array}{lccccc}\text { Predictor } & \text { Coef } & \text { StDev } & \text { T } & \text { P } & \text { VIF } \\ \text { Constant } & 0.60264 & 0.07622 & 7.91 & 0.000 & \\ \text { date } & -0.006068 & 0.001111 & -5.46 & 0.000 & 1.0 \\ \text { cap } & -0.00002313 & 0.00000593 & -3.90 & 0.001 & 1.0 \\ \text { ne } & -0.007811 & 0.002564 & -3.05 & 0.005 & 1.0 \\ \text { S }=0.006251 & \text { R-Sq }=67.7 \% & \text { R-Sq }(\operatorname{adj})=64.2 \%\end{array}$


Analysis of Variance

$\begin{array}{llllll}\text { Source } & \text { DF } & \text { SS } & \text { MS } & \text { F } & \text { P }\end{array}$

$\begin{array}{llllll}\text { Regression } \quad 3 & 0.00229188 & 0.00076396 & 19.55 & 0.000\end{array}$

Residual Error 280.001094130 .00003908

Total $\quad 310.00338600$

Best Subsets Regression

Response is $1 / \mathrm{ln}$ y

Adj.

Vars R-Sq R-Sq C-p s e p e

$\begin{array}{llllll}1 & 39.7 & 37.6 & 24.3 & 0.0082526 & \mathrm{X}\end{array}$

$1 \quad 18.4 \quad 15.6 \quad 42.70 .0095993 \quad \mathrm{X}$

$\begin{array}{lllllll}2 & 57.0 & 54.0 & 11.3 & 0.0070874 & X X\end{array}$

$\begin{array}{llllllll}2 & 50.1 & 46.7 & 17.2 & 0.0076304 & X & X\end{array}$

$\begin{array}{llllll}3 & 67.7 & 64.2 & 4.0 & 0.0062511 & \text { X X X }\end{array}$

Stepwise Regression: 1/lny versus sdate, scap, sne

Alpha-to-Enter: 0.15 Alpha-to-Remove: 0.15

Response is $1 /$ lny on 3 predictors, with $\mathrm{N}=32$

\begin{tabular}{|c|c|c|c|}
\hline Step & 1 & 2 & 3 \\
\hline Constant & 0.6100 & 0.6232 & 0.6026 \\
\hline sdate & -0.0065 & -0.0064 & -0.0061 \\
\hline T-Value & -4.44 & -5.10 & -5.46 \\
\hline P-Value & 0.000 & 0.000 & 0.000 \\
\hline scap & & $00002-0$. & .00002 \\
\hline T-Value & & -3.42 & -3.90 \\
\hline P-Value & & 0.002 & 0.001 \\
\hline sne & \multicolumn{3}{|c|}{-0.0078} \\
\hline T-Value & \multicolumn{3}{|c|}{-3.05} \\
\hline P-Value & \multicolumn{3}{|c|}{0.005} \\
\hline $\mathrm{S}$ & 0.00825 & 0.00709 & 0.00625 \\
\hline $\mathrm{R}-\mathrm{Sq}$ & 39.66 & 56.98 & 67.69 \\
\hline R-Sq(adj) & ) $\quad 37.65$ & 54.01 & 64.22 \\
\hline Mallows & C-p 24. & 11.3 & 4.0 \\
\hline
\end{tabular}

\section{DISCUSSION}

After conducting the above regression methods and the stepwise regressions we can conclude the following results:

\section{A. Over All F-test :}

$\mathrm{HO}:$ all $\mathrm{Bi}=$ zero

$\mathrm{Ha}:$ at least one $\mathrm{Bi}$ not $=$ zero

p-value $=0$, Reject the null hypothesis

therefore at least one Bi not equal to zero . the model stands.

B. Predictors :

Looking at Stepwise Regression, Best Subsets Regression and the P-value of each predictor, and its corresponding seq ss.

We can say that ;

All p-values are $\approx$ zero....so we are good.

C. Best Subsets :

Looks like we won't be able to get rid of any of them, or else bias problems will occur. BUT there are 2 obvious outliers in the residual plot... so we need to run the test for outlier. 

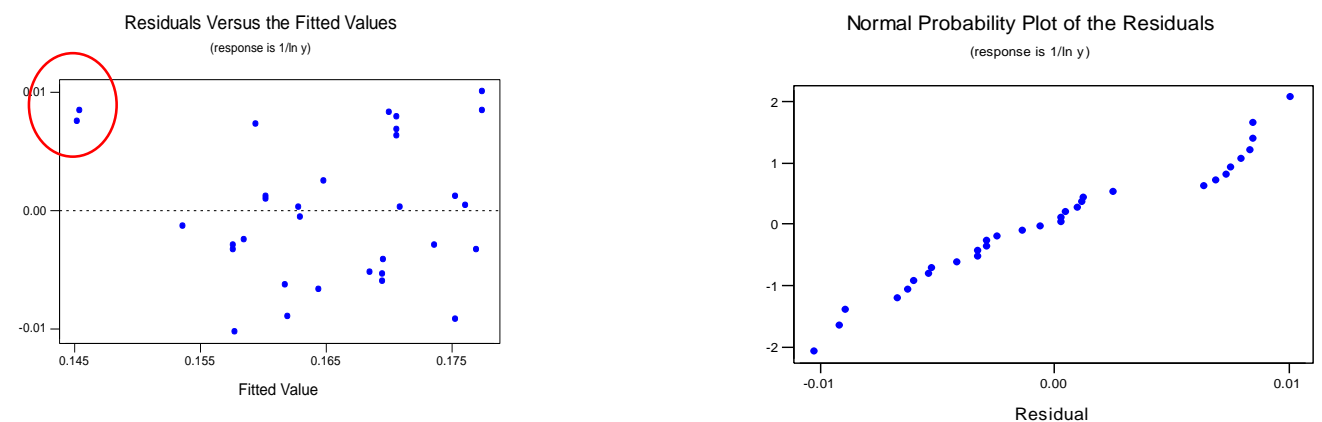

Fig. 10 Normal probability and residual vs fitted values plots after factors elimination

Therefore, from Fig 10 we have to conduct the outlier tests to see whether these points should be eliminated or not. The outlier test will be as follow:

\section{Hat matrix indicator}

Index $=2 \mathrm{p} / \mathrm{n}=0.1875$

Therefore this subjects that cases 22 and 26 are highly influential

2. Cook's Distance :

The cases 22 and 26 have high cook's distance, this supports that they are highly influential

\section{DFFITS :}

Index $=2 \operatorname{sqrt}(\mathrm{p} / \mathrm{n})=0.61$

The cases 22 and 26 also have high DFFITS, this supports that they are highly influential

\section{DFBitas Appendix ( C ):}

The cases 22 and 26 also have high Effect on the parameters b0, b1, and b3.

When we went back to the data collected it showed that the two nuclear power stations had different characteristics than the rest and that it would be wise to remove them from the data set.

The Model after Removing case 26:
Table 1: Outlier test

\begin{tabular}{|c|c|c|c|}
\hline & Hat Mat & COOKS & DFITS \\
\hline 1 & 0.142463 & 0.000109 & 0.02048 \\
\hline 2 & 0.140142 & 0.042856 & -0.41443 \\
\hline 3 & 0.140142 & 0.034836 & -0.37229 \\
\hline 4 & 0.196627 & 0.020828 & -0.28517 \\
\hline 5 & 0.196627 & 0.016356 & -0.25238 \\
\hline 6 & 0.231088 & 0.00021 & 0.02843 \\
\hline 7 & 0.045655 & 0.022448 & 0.30464 \\
\hline 8 & 0.164355 & 0.016177 & -0.25128 \\
\hline 9 & 0.042027 & 0.007902 & -0.17687 \\
\hline 10 & 0.131365 & 0.088482 & -0.61026 \\
\hline 11 & 0.105586 & 0.007065 & -0.16579 \\
\hline 12 & 0.125995 & 0.05682 & 0.48191 \\
\hline 13 & 0.120704 & 0.083456 & -0.59373 \\
\hline 14 & 0.091118 & 0.00069 & 0.05161 \\
\hline 15 & 0.047048 & 0.002109 & 0.09046 \\
\hline 16 & 0.044061 & 0.00528 & -0.14389 \\
\hline 17 & 0.143094 & 0.002175 & -0.09168 \\
\hline 18 & 0.120704 & 0.001423 & 0.07413 \\
\hline 19 & 0.116337 & 0.100083 & -0.65808 \\
\hline 20 & 0.091118 & 0.001079 & 0.06456 \\
\hline 21 & 0.049932 & 0.015735 & -0.2518 \\
\hline 22 & 0.273792 & 0.238601 & 1.00588 \\
\hline 23 & 0.084868 & 0.025216 & -0.31811 \\
\hline 24 & 0.118175 & 0.005791 & -0.14991 \\
\hline 25 & 0.233622 & 0.000818 & -0.05617 \\
\hline 26 & 0.325807 & 0.261149 & 1.04478 \\
\hline 27 & 0.098258 & 0.078782 & 0.58213 \\
\hline 28 & 0.099677 & 0.000171 & 0.02568 \\
\hline 29 & 0.060452 & 0.017759 & 0.26704 \\
\hline 30 & 0.060452 & 0.02067 & 0.28907 \\
\hline 31 & 0.098258 & 0.055546 & 0.4807 \\
\hline 32 & 0.060452 & 0.027888 & 0.33862 \\
\hline
\end{tabular}



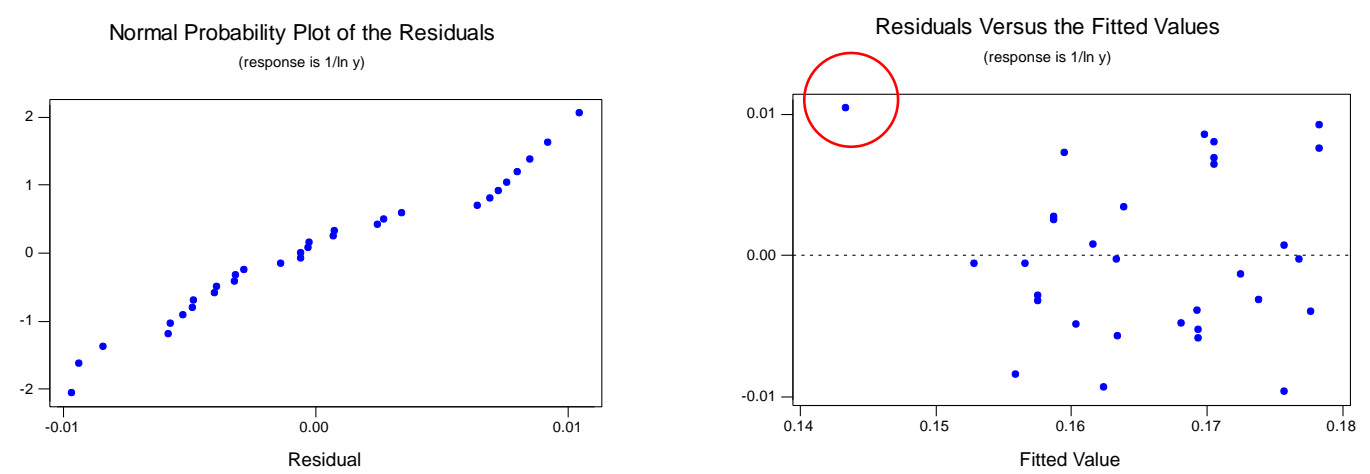

Fig. 11 Normal probability and residual vs fitted values plots after eliminating case 22

We can see that we actually have a better normal probability plot, and we only have one outlier now in the residual plot. Note that the model is still significant. The Model after Removing case 22 will be as shown in Fig. 11 We can see that we actually have a better normal probability plot, and we only have no outliers in the residual plot. Note that the model is still significant, and with even more overall F-statistic value and more R-sq (adj)
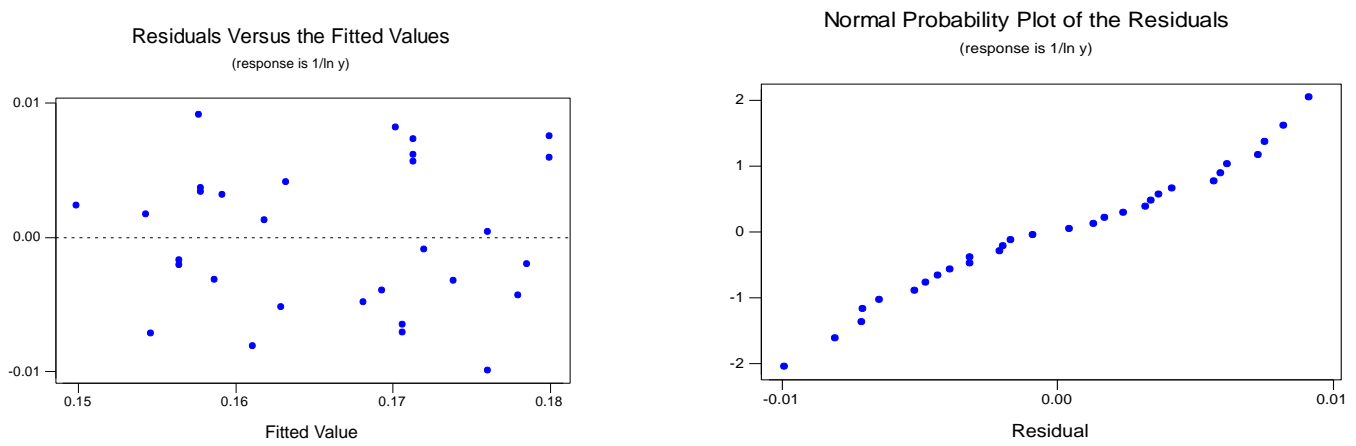

Fig. 12 Normal probability and residual vs fitted values plots after eliminating case 22 and 26

And therefore, after removing cases 22 and 26 the regression equation will be as follow:

$1 /$ ln $y=0.760-0.00833$ date -0.000027 cap -0.00860 ne

$\begin{array}{lclllll}\text { Predictor } & \text { Coef } & \text { StDev } & \text { T } & \text { P V VIF } & \\ \text { Constant } & 0.75995 & 0.09067 & 8.38 & 0.000 & \\ \text { date } & -0.008325 & 0.001313 & -6.34 & 0.000 & 1.0 \\ \text { cap } & -0.00002709 & 0.00000575 & -4.71 & 0.000 & 1.0 \\ \text { ne } & -0.008596 & 0.002469 & -3.48 & 0.002 & 1.0\end{array}$

$\mathrm{S}=0.005718 \quad \mathrm{R}-\mathrm{Sq}=72.3 \% \quad \mathrm{R}-\mathrm{Sq}(\mathrm{adj})=69.1 \%$

Analysis of Variance

\begin{tabular}{|c|c|c|c|c|c|}
\hline Source & $\mathrm{DF}$ & SS & MS & $\mathrm{P}$ & \\
\hline Regress & & 30.00221929 & 0.00073976 & 22.63 & 0.000 \\
\hline Residua & Error & 260.00085008 & $\begin{array}{ll}8 & 0.00003270\end{array}$ & & \\
\hline Total & 29 & 0.00306937 & & & \\
\hline Source & DF & Seq SS & & & \\
\hline date & 10.00 & 106928 & & & \\
\hline cap & 10.00 & 075374 & & & \\
\hline ne & 10.00 & 039628 & & & \\
\hline
\end{tabular}




\section{SUMMARY AND RESULTS}

From the above we can conclude that the cost of a future power plant can be estimated using,

- date: The date on which the construction permit was issued. The data are measured in years since January 11990 to the nearest month.

- cap: The net capacity of the power plant (MWe).

- ne: A binary variable where 1 indicate that plant was constructed in the north-east region of US.

And this can be done using the regression equation

$$
1 / \ln \text { Cost }=0.760-0.00833 \text { date }-0.000027 \text { cap }-0.00860 \text { ne }
$$

Or in other words, the Cost of a future power plant can be calculated from the following relationship:

$$
\text { Cost }=\exp (1 /(0.760-0.00833 \text { date }-0.000027 \text { cap }-0.00860 \text { ne }))
$$

\section{CONCLUSIONS}

In this paper the multiple regression models to estimate the cost of constructing Light Water Reactor (LWR) plants has been developed using previous data. Since the goal would be minimizing the cost of construction, and through the model we see that the cost would increase with the increase of Date, Capacity and the location (in the North-East region of the US). So based on our model we can recommend that the construction permit should be issued as soon as possible, the net capacity of the power plant should be kept with the expected loads and not to construct in the North-East region of the US. In the future, the proposed regression model can be enhanced by considering other factors that might affect the cost of construction. Another promising avenue for future research is to apply the proposed regression model to similar application.

\section{REFERENCES}

[1] Cox, D.R and Snell, E.J. (1981) Applied Statistics: Principles and Examples. Chapman and Hall (Source of the data)

[2] B.R. Sehgal, "Stabilization and termination of severe accidents in LWRs", Nucl. Eng. Design 236, 1941 (2006).

[3] B R Sehgal, Light Water Rector (LWR) Safety, Nuclear Engineering and Technology, VOL.38 NO.8, 2006

[4] Babyak MA. What you See May not be What you Get: A Brief, Nontechnical Introduction to Over Fitting in Regression-Type Models. Psychosom Med 2004;66:411-21.

[5] Derksen S, Keselman H. Backward, Forward and Stepwise Automated Subset Selection Algorithms: Frequency of Obtaining Authentic and Noise Variables, Br J Math Stat Psychol 1992;45:265-82.

[6] Steyerberg EW, Eijkemans MJ, Harrell FE Jr., et al. Prognostic Modeling with Logistic Regression Analysis: in Search of a Sensible Strategy in Small Data Sets. Med Decis Making 2001;21:45-56.

[7] Kleinbaum D, Klein M. Logistic regression. 2nd edn. New York: Springer-Verlag, 2002.

[8] S Abdul-Wahab, Charles S. Bakheit, Saleh M. Al-Alawi, Principal Component and Multiple Regression Analysis in Modeling of Ground-Level Ozone and Factors Affecting its Concentrations, Environmental Modeling \& Software, Volume 20, Issue 10, October 2005, Pages 1263-1271

[9] H Cordell and D Clayton, A Unified Stepwise Regression Procedure for Evaluating the Relative Effects of Polymorphisms within a Gene Using Case/Control or Family Data: Application to HLA in Type 1 Diabetes, $A J H G$, V 70, Issue $12002,124-141$

[10] Neter, Kutner, Nachtsheim, Wasserman Applied Linear Regression Models, 3rd Edition. 1996 